\title{
Use of BioFire FilmArray gastrointestinal PCR panel associated with reductions in antibiotic use, time to optimal antibiotics, and length of stay
}

Daisy Torres-Miranda, Hana Akselrodº, Ryan Karsner, Alessandra Secco, Diana Silva-Cantillo, Marc O. Siegel, Afsoon D. Roberts and Gary L. Simon

\begin{abstract}
Background: Rapid and accurate diagnostic tools are needed for appropriate management of infectious diarrhea. Methods: We evaluated the impact of the introduction of rapid multiplex PCR testing using the FilmArray gastrointestinal (GI) panel (BioFire Diagnostics, LLC, Salt Lake City, UT) at our institution, and compared the results to those of standard stool cultures.

Results: The most common pathogens detected by the FilmArray Gl panel were Clostridium difficile (55.0\%), Campylobacter species (20.9\%), Salmonella species (12.4\%), and Shigella/EIEC species (12.4\%). Rates of reproducibility in stool culture for these pathogens ranged from 56.3 to $77.8 \%$. Co-detection of two or more organisms was common (24.2\%), most commonly involving EPEC, EAEC, ETEC, and STEC. The time from arrival in the Emergency Department to discharge or admission to the hospital was unchanged after the introduction of FilmArray Gl panel, but length of hospital stay was shorter ( 3 vs. 7.5 days, $p=0.0002)$ for the FilmArray group. The time to empiric antibiotics did not differ significantly, but optimal antibiotics were started earlier after introduction of the FilmArray Gl panel (hospital day 1 vs. $2, p<0.0001$ ). More patients were discharged without antibiotics after introduction of the FilmArray Gl panel (14.0\% vs. $4.5 \% ; p<0.001)$.
\end{abstract}

Conclusion: Our results demonstrate that the FilmArray Gl panel is an important tool for improving both patient care and antibiotic stewardship, despite the tendency for positive results with multiple pathogens.

Keywords: Gastrointestinal, FilmArray, Multiplex PCR, Diarrhea, Antibiotics, Stewardship

* Correspondence: hakselrod@gwu.edu

Division of Infectious Diseases, George Washington University School of Medicine and Health Sciences, 2150 Pennsylvania Avenue NW, Washington, DC 20037, USA

(c) The Author(s). 2020 Open Access This article is licensed under a Creative Commons Attribution 4.0 International License, which permits use, sharing, adaptation, distribution and reproduction in any medium or format, as long as you give appropriate credit to the original author(s) and the source, provide a link to the Creative Commons licence, and indicate if changes were made. The images or other third party material in this article are included in the article's Creative Commons licence, unless indicated otherwise in a credit line to the material. If material is not included in the article's Creative Commons licence and your intended use is not permitted by statutory regulation or exceeds the permitted use, you will need to obtain permission directly from the copyright holder. To view a copy of this licence, visit http://creativecommons.org/licenses/by/4.0/ The Creative Commons Public Domain Dedication waiver (http://creativecommons.org/publicdomain/zero/1.0/) applies to the data made available in this article, unless otherwise stated in a credit line to the data. 


\section{Background}

Infectious gastroenteritis remains a common cause of morbidity and mortality worldwide [1-3]. In the United States, an estimated 179 million episodes of diarrheal illness are estimated to occur each year, resulting in nearly 500,000 hospitalizations and 5000 deaths. The economic burden of Emergency Department (ED) visits for diarrheal illnesses alone in the USA has been estimated at \$580 million per year [4-7]. Stool cultures have been the standard diagnostic tool for determining the microbial etiology of suspected bacterial infectious diarrhea. However stool cultures are time consuming, costly, have low positive yield, and their use by physicians can be inconsistent [8-11]. Rapid and accurate diagnostic tools are needed for appropriate management of infectious gastroenteritis. Commercially available multiplex polymerase chain reaction (PCR) testing panels may improve patient care and hospital workflow by allowing clinicians to choose the appropriate antimicrobials, or to avoid them if they are not indicated, aiding antibiotic stewardship and reduction of harm such as infection with C.lostridium difficile [12-15].

The FilmArray Gastrointestinal (GI) PCR Panel (BioFire Diagnostics, Salt Lake City, UT, USA) received FDA approval in 2014 and can identify 22 bacterial, viral, and protozoan pathogens associated with gastroenteritis directly from stool samples within approximately $1 \mathrm{~h} \mathrm{[16].} \mathrm{The} \mathrm{proposed}$ advantage of this test is the rapid pathogen identification thereby expediting appropriate treatment, including initiation or discontinuation of therapeutic agents and isolation precautions [17]. Currently, the question of whether the use of this test leads to quantifiable improvements in patient care over that of standard stool cultures is unresolved [18, 19]. We evaluated the impact of adding FilmArray GI panel testing to the standard management of patients presenting to the Emergency Department (ED) with suspected infectious diarrhea over an 18-month observation period (12 months prior to the introduction of GI BioFire, and 6 months after). This was a retrospective chart review performed with the specific aims of defining the reproducibility of FilmArray GI panel results in comparison to standard stool cultures, as well as time to disposition from the emergency department, duration of hospitalization, duration of antibiotic use, and time to optimal therapy, in a real-world setting. This study helps establish the role of multiplex PCR in the management of patients in the ED and hospital settings, and should be of interest to health care practitioners and leaders seeking to make decisions regarding the use of these testing modalities.

\section{Methods}

\section{Patient population and data collection}

We reviewed the electronic medical records for 300 adults seen at the George Washington University Hospital (GWUH) ED for a diagnosis of infectious diarrhea (identified by ICD-9/ICD-10 codes) from July 2015 through December 2016. FilmArray GI panel testing became available in May 2016. Variables abstracted into the study database included the FilmArray GI panel and stool culture results, other microbiology results (e.g. blood cultures), ED arrival and disposition times, duration of hospitalization, initiation of empiric and of optimal therapy, duration of final prescribed therapy, and outcome of the hospitalization. "Optimal antibiotics" were defined as those having established activity against the bacterial pathogen in question, with clinically appropriate selection, dosing, and duration of treatment in accordance with published guidelines and professional standard of care, as confirmed upon review by the authors. Infectious diarrhea was defined as three or more loose stools in a 24-h period accompanied by other gastrointestinal symptoms such as nausea, vomiting, abdominal cramps, or an oral temperature $\geq 38{ }^{\circ} \mathrm{C}$. Patients for whom either the stool culture or FilmArray GI panel were positive but who did not meet the above clinical criterial for infectious diarrhea at the time of presentation were excluded from the analysis.

\section{Laboratory methods}

The FilmArray GI panel includes the following bacterial pathogens: Campylobacter (jejuni, coli, and upsaliensis), Clostridium difficile toxin A/B, Plesiomonas shigelloides, Salmonella spp., Yersinia enterocolitica, Vibrio (parahaemolyticus, vulnificus, and cholerae), Enteroaggregative Escherichia coli (EAEC), Enteropathogenic E. coli (EPEC), Enterotoxigenic E. coli (ETEC), Shiga-like toxin-producing E. coli (STEC) stx1/stx2, E. coli O157, and Shigella/Enteroinvasive E. coli (EIEC) [16, 17]. Shigella and EIEC could not be differentiated on the basis of the FilmArray GI panel alone without confirmatory cultures. FilmArray GI panel results that were positive for only viral or parasitic pathogens were excluded, as this study focused on the antibiotic management of bacterial diarrhea. All stool samples where bacterial pathogens were detected by the FilmArray GI panel were reflexed to standard stool cultures. Stool cultures were able to identify the following species: Yersinia, Shigella, Salmonella, Vibrio, Campylobacter, and E. coli O157. Stool cultures containing only normal fecal flora, including all E. coli serotypes other than O157, were reported as negative. Testing for $C$. difficile was done by the toxin A/ B EIA and PCR two-tier approach.

\section{Data analysis}

Univariate and bivariate statistics were calculated for the variables of interest using Statistical Analysis Software (SAS, Cary, NC, software version 9.4). Assumptions of normal distribution were made initially in the study design phase. In the course of the study it became apparent that the two comparison groups were unequally affected by miscoding and chart duplication, resulting in unequal 
samples size and variances; therefore, Welch's rather than Student's t-test was used where appropriate. Where univariate analysis demonstrated non-Normal distribution of the outcome variables, nonparametric analysis was conducted using the Wilcoxon rank-sum (MannWhitney U-test) method. Where both comparison groups included up to 50 observations, exact two-sided test statistics were calculated; otherwise the normal approximation with continuity correction of 0.5 was applied. The study procedures were approved by the GW University Office of Human Research Institutional Review Board.

\section{Results}

Of 300 patient records reviewed for this study, 177 were seen after the introduction of FilmArray GI panel ("FilmArray group") and 123 were seen before ("pre-FilmArray" or historical control group). Rates of patient exclusion differed between the two groups (Fig. 1). The most common reason for exclusion was lack of diarrhea observed during the hospital stay but not at the time of initial arrival/evaluation (42/177 in the FilmArray group, 42/123 in the preFilmArray group), chart duplication (2/177 in the FilmArray group, 30/123 in the pre-FilmArray group), and inclusion of non-infectious diarrhea cases in the initial search results (2/177 in the FilmArray group, 10/123 in the pre-FilmArray group). One hundred twenty-nine of $177 \mathrm{pa}-$ tients $(73 \%)$ in FilmArray group met inclusion criteria while only 41 of 123 patients (33\%) in the pre-FilmArray comparison group met inclusion criteria. This led to an adjustment of the statistical approach as noted in Methods. The resultant groups differed in composition by gender, age, and rates of $C$. difficile infection (CDI) (Table 1).

Of the 129 patients tested with the FilmArray GI panel, C. difficile was the most frequently detected organism and was found in 71 (55.0\%) samples. Campylobacter was detected in 27 (20.9\%) of samples. Salmonella was detected in $16(12.4 \%)$ patients, two of whom were bacteremic. BioFire detected Shigella/EIEC in 16 (12.4\%) patients. EPEC, EAEC, ETEC, and STEC were the most common codetected organisms, found in 18 (14.0\%) of patients, always in combination with other pathogens. There were three cases of $E$. coli $\mathrm{O} 157$ detected, but only one was confirmed by culture. There was one case of Vibrio parahemolyticus detected which was confirmed in culture. Two or more pathogens were detected by FilmArray GI panel in 31 (24.2\%) of cases, as illustrated (Fig. 2). Confirmation by culture ranged from $56.3 \%$ for Campylobacter to $77.8 \%$ for Shigella (Fig. 3), but was limited by low rates of confirmatory cultures which were only ordered for 40 of the 68 non-C. difficile patients.

Median time from ED arrival to overall disposition was $7 \mathrm{~h}$ (IQR: $5-10)$ both for the FilmArray group and the pre-FilmArray group $(p=0.6052)$. This was not significantly different among those patients who were admitted to the hospital or those discharged from the ED. The rate of admission to the hospital was $62.8 \%$ in the FilmArray group and $87.8 \%$ in the pre-FilmArray group $(p<0.0001)$. The hospital length of stay (LOS) ranged from 1 to 60 days overall, with median LOS of 3 (IQR: $2-6)$ days in the FilmArray group and 7.5 (IQR: 4-10) days in the control group $(p=0.0002)$.

Median time to initiation of empiric antibiotics did not differ significantly between the two groups, but the time to optimal therapy (defined as the antibiotic prescribed at the time of hospital discharge) was shorter in the FilmArray group ( 1 vs. 2 days, $p=0.0117$ ). The proportion of patients for whom the initial antibiotics choice was not changed for discharge was $47.3 \%$ in the FilmArray group vs. $22.0 \%$ in the control group $(p<$ 0.0001). Patients discharged without antibiotics (either not started on antibiotics, or started and then discontinued prior to discharge) accounted for $14.0 \%$ of the

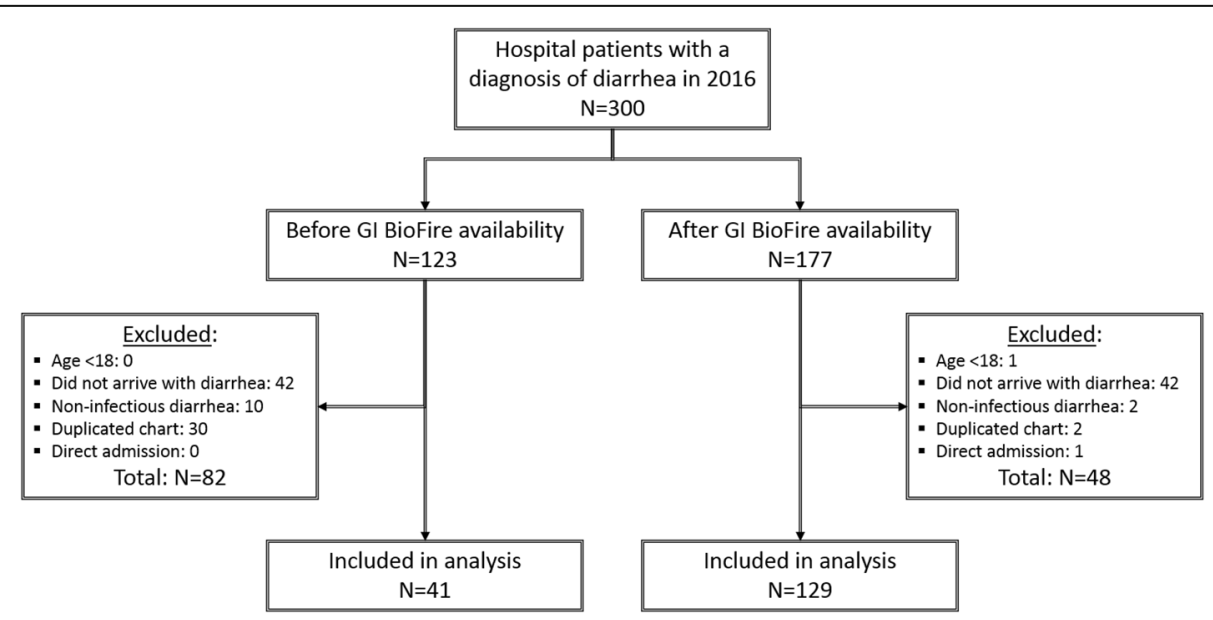

Fig. 1 Patient inclusion and exclusion flowchart 
Table 1 Characteristics of patients with suspected infectious diarrhea evaluated before and after introduction of FilmArray GI PCR

\begin{tabular}{|c|c|c|c|c|}
\hline Characteristic & Overall & FilmArray group & Pre-FilmArray group & $\boldsymbol{P}$-value* \\
\hline \multicolumn{5}{|l|}{ Total } \\
\hline N & 170 & 129 & 41 & - \\
\hline \multicolumn{5}{|l|}{ Sex } \\
\hline Male - N (\%) & 79 (46.5\%) & $65(50.4 \%)$ & $14(34.1 \%)$ & 0.0120 \\
\hline \multicolumn{5}{|l|}{ Age } \\
\hline Years - mean \pm std. dev. & $47.4 \pm 18.4$ & $45.6 \pm 18.4$ & $56.7 \pm 17.9$ & 0.0011 \\
\hline \multicolumn{5}{|l|}{ Prior history of CDI } \\
\hline Prior C. difficile documented in chart - N (\%) & $22(12.9 \%)$ & $13(10.1 \%)$ & $9(22.0 \%)$ & $<0.0001$ \\
\hline \multicolumn{5}{|l|}{ CDI diagnosis (by ICD-9/ICD-10 code) } \\
\hline Clostridium difficile enteritis/colitis - N (\%) & $102(60.0 \%)$ & $68(52.7 \%)$ & 34 (82.9\%) & $<0.0001$ \\
\hline \multicolumn{5}{|l|}{ Time to overall disposition from ED } \\
\hline Hours - median [Q1,Q3] & $7[5,10]$ & $7[5.5,10]$ & $7[4,10.5]$ & 0.6052 \\
\hline \multicolumn{5}{|l|}{ Time to discharge from ED } \\
\hline Hours - median [Q1,Q3] & $7[5,10]$ & $7.5[5,10]$ & $7[6,15]$ & 0.5618 \\
\hline \multicolumn{5}{|l|}{ Time to admission to hospital } \\
\hline Hours - median [Q1,Q3] & $7[5,10]$ & $7[6,10]$ & $7[4,10]$ & 0.4542 \\
\hline \multicolumn{5}{|l|}{ Hospital admission } \\
\hline Admitted from ED to hospital - N (\%) & $117(68.8 \%)$ & $81(62.8 \%)$ & $36(87.8 \%)$ & $<0.0001$ \\
\hline \multicolumn{5}{|l|}{ Hospital length of stay (LOS) } \\
\hline Days - median [Q1,Q3] & $4[2,8]$ & $3[2,6]$ & $7.5[4,10]$ & 0.0002 \\
\hline \multicolumn{5}{|l|}{ Day on which empiric antibiotics started } \\
\hline Days - median [Q1,Q3] & $1[1,1]$ & $1[1,1]$ & $1[1,1]$ & 0.3298 \\
\hline \multicolumn{5}{|l|}{ Day on which optimal antibiotics started } \\
\hline Days - median [Q1,Q3] & $2[1,2]$ & $1[1,2]$ & $2[1,3]$ & 0.0117 \\
\hline \multicolumn{5}{|l|}{ Optimal antibiotics prescribed on first try } \\
\hline Discharged with initial antibiotics - N (\%) & $70(41.2 \%)$ & $61(47.3 \%)$ & $9(22.0 \%)$ & $<0.0001$ \\
\hline \multicolumn{5}{|l|}{ Discharged without antibiotics } \\
\hline Antibiotics not prescribed or discontinued - N (\%) & $20(11.8 \%)$ & $18(14.0 \%)$ & $2(4.9 \%)$ & $<0.0001$ \\
\hline
\end{tabular}

* Welch's t-test or Wilcoxon ranked-sum test (Mann-Whitney U-test) for continuous variables, Chi-squared test for categorical variables

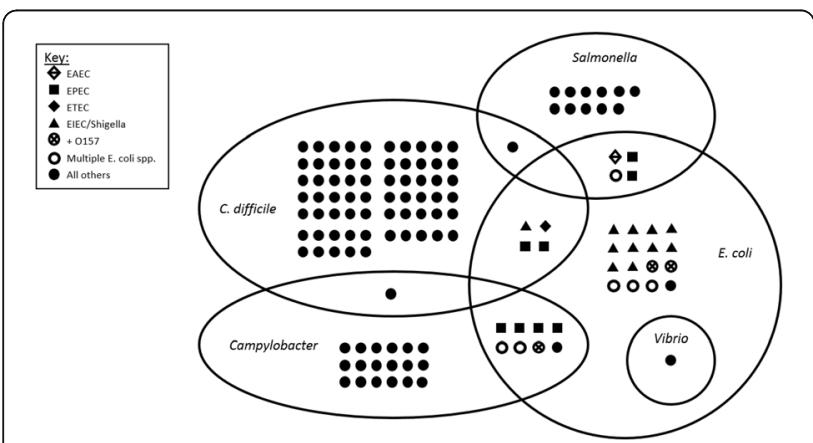

Fig. 2 FilmArray GI PCR results as reported on initial stool samples of 129 patients with suspected infectious diarrhea. E. coli pathotypes are coded according to the key below, including where two or more pathotypes were detected (o); the three samples in which $E$. coli $\mathrm{O} 157$ was detected or co-detected are shown (see text)
FilmArray group and $4.9 \%$ of the pre-FilmArray group. Among the 28 patients whose FilmArray GI panel results were confirmed by culture, $28.6 \%$ were discharged without antibiotics. All patients diagnosed with CDI received therapy with either metronidazole or oral vancomycin. The most frequent outpatient antibiotic prescribed to patients without CDI in both groups was ciprofloxacin.

\section{Discussion}

The potential of using multiplex PCR testing for the diagnosis and management of infectious diarrheal illness has been extensively documented in a variety of settings [13-15, 17, 20, 21], but so has its potential for yielding results of unclear clinical significance, including falsepositives and multiple positives per sample [18, 19, 22, 23]. This tendency is inherent in the technology, and is a result 


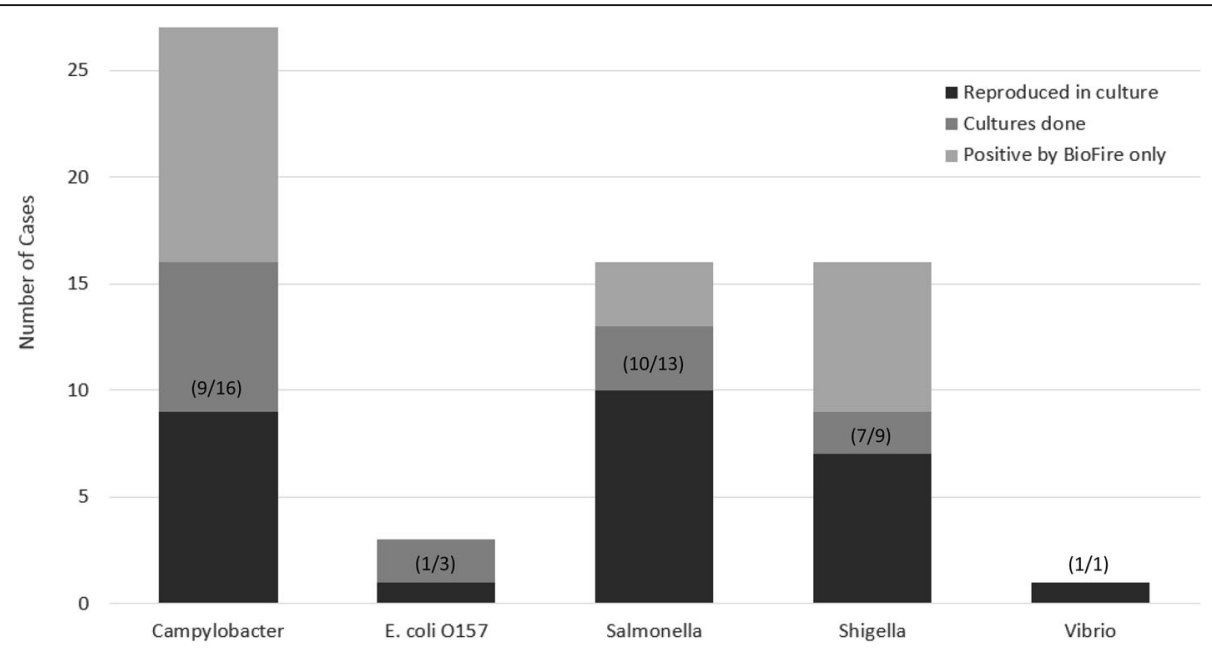

Fig. 3 Reproducibility of FilmArray GI PCR findings in culture, by pathogen. Proportion of confirmatory cultures positive for the target organism given in parentheses

of its reliance on the amplification of genetic loci which may persist in the gut during asymptomatic carriage, or be transferred horizontally between enteric bacteria, particularly $E$. coli pathotypes and other enteric bacteria [16, 17, 22, 24, 25]. In clinical use, this creates a conflict between the desire to expedite diagnostic results in patients with infectious gastroenteritis, and the potential to over-treat non-pathological results.

Co-detection of multiple pathogens, particularly multiple E. coli pathotypes, was common in our study. This is similar to findings in prior studies of multiplex PCR panels for gastrointestinal sample analysis, in which codetections were reported in $16-28 \%$ of positive samples [22-26]. The FilmArray GI panel is noted to be more prone to detecting mixed infections than the Luminex xTAG system (Luminex Corporation, Austin, TX), the only other FDA-approved multiplex PCR gastrointestinal panel [22]. Whether such detections represent true coinfections of viable organisms or merely colonization cannot be determined based on these tests alone, and decisions regarding treatment continues to be a clinical issue. Standard bacterial cultures continue to have a key role in confirming PCR diagnoses, but published rates of successful culture confirmation of multiplex PCR findings range widely. In one meta-analysis, culture confirmation was 63.9\% for Campylobacter (CI: 39.8-84.9\%), 48.4\% for Salmonella (CI: 27.8-69.3\%), 73.4\% for Shigella (CI: 38.197.1\%), and $75.0 \%$ for E. coli O157 (CI: 50.9-91.3\%); overall, the PCR methods detected approximately 1.5 times more pathogens compared to standard stool cultures [18].

Overestimation of C. difficile infections is welldocumented risk of using PCR-based diagnostics. In one study of the FilmArray GI panel, only $43 \%$ of samples where C. difficile was detected were subsequently found to produce detectable toxin by enzyme immunoassay (EIA) [27]. In our study we found that physicians did not consistently order a confirmatory EIA in patients who had $C$. difficile detected by the FilmArray GI panel. This has since been addressed with quality improvement interventions in our institution, and a consistent two-step reflex testing algorithm instituted. Physicians have also been educated about refraining from ordering the GI multiplex panel when a high pre-test suspicion for CDI exists. Additional aggressive institutional and regional efforts in antibiotic stewardship and infection prevention aimed at reducing CDI incidence have been ongoing. It is possible that these efforts contributed to the difference in LOS and antibiotics use we observed, though the relatively short period of time captured (2015-2016) makes it less likely that such changes in practice or other secular trends (such as the tendency towards shorter LOS overall) entirely account for our findings.

Several recent multi-center studies have compared the FilmArray GI panel to other PCR assays or conventional culture methods with mixed results. While initial studies showed high sensitivity and specificity of FilmArray GI panel when compared to reference methods [16, 17, 22], other real-world studies have found more variation in the rates of pathogen detection and reproducibility, depending on the practices of specific centers [23-26]. The use of the FilmArray GI panel has implications regarding other aspects of patient care, including use of hospital resources and infection control. Beal et al. found a modest reduction in LOS, days of antibiotics, abdominal imaging studies, and costs per patient associated with the use of the FilmArray GI panel; however, in that study the FilmArray GI panel results were not communicated to physicians in a timely manner, likely reducing their impact on management [28]. In a cost-benefit analysis, the use of the Luminex xTAG gastrointestinal panel was associated with increased diagnostic costs but break- 
even points and cost savings could be realized contingent on using the test results to reduce isolation days [29]. In practice, physicians frequently fail to follow up on these results with appropriate adjustment of isolation status [30]. Some experts favor restricting the use of multiplex PCR panels to initial evaluations or to patients with severe symptoms in order to improve costeffectiveness [31, 32].

In our study, the introduction of FilmArray GI panel testing did not appear to affect overall time to disposition (either hospital admission or discharge from the ED), or time to empiric antibiotics. These findings are not altogether surprising, as initial management is more likely to be driven by clinical factors, including patient characteristics and illness severity, than by the findings of a single diagnostic test. Administrative and logistical factors might also affect the time to admission or discharge. Overall LOS was significantly shorter after the introduction of FilmArray GI panel testing, as was the time to initiate appropriate optimal antibiotics. These findings suggest that the use of the FilmArray GI panel as part of the initial evaluation of patients with acute diarrhea of suspected infectious etiology may result in cost savings.

The role of multiplex PCR in antibiotic stewardship remains mixed. On one hand, a positive result in the absence of proper clinical interpretation may lead to unnecessary treatment. The high proportion of patients in our study who received treatment for organisms found on the FilmArray GI panel alone (not confirmed by culture results) may speak to the propensity of physicians to treat reflexively based on reported test results. On the other hand, in the era of stool culture-based diagnosis alone, only $37 \%$ of patients in our sample were started on the optimal medication right away, whereas after the introduction of the FilmArray GI panel this increased to $52 \%$, and optimal treatment occurred sooner in their hospital course. By enabling physicians to avoid starting antibiotics where not indicated (e.g. viral enteritis or certain E. coli types), and targeting therapy to pathogen earlier in the treatment course, this methodology can support the aims of antibiotic stewardship.

Our study has several limitations. The number of patients meeting inclusion criteria was unequally affected by miscoding (i.e. patient charts initially selected based on diagnostic codes, which were then excluded), resulting in an especially low number in the control group, and compromising the overall statistical power. By its very nature, a retrospective review is dependent upon correct coding, and it is likely that some patients were incorrectly diagnosed and their charts could not be accessed. The high prevalence of CDI-related diagnoses in our pre-FilmArray group may reflect a sampling bias in which cases of suspected infectious diarrhea were not coded as such in the absence of a specific pathogen (in turn reflecting the low diagnostic yield of stool cultures) and thus overlooked when screening patient charts for inclusion in the study. We adjusted for these issues to the best of our ability by using more conservative statistical methods.

\section{Conclusions}

Clinical judgment combined with multiplex PCR and confirmatory stool cultures can provide an approach to infectious gastroenteritis that is both rapid and accurate. The increased laboratory costs associated with this approach should be offset by the reduction in inappropriate antibiotic administration and decrease in LOS. Further research is needed to understand the optimal use and interpretation of multiplex PCR methods in the diagnosis of infectious diarrhea.

\section{Abbreviations \\ CDI: Clostridium difficile infection; EAEC: Enteroaggregative E. coli; \\ ED: Emergency Department; EIEC: Enteroinvasive E. coli; \\ EPEC: Enteropathogenic E. coli; ETEC: Enterotoxigenic E. coli; \\ Gl: Gastrointestinal; GW OHR IRB: George Washington University Office of Human Research Institutional Review Board; GWUH : George Washington University Hospital; LOS: Length of stay; PCR: Polymerase chain reaction; STEC: Shiga toxin-producing E. coli}

\section{Acknowledgments}

The authors give their special thanks to Dr. John Keiser, Director of the GWUH Microbiology Lab, and Mr. Madhi Moshgriz, Microbiology Technical Specialist, for laboratory materials and testing provided for this study.

\section{Authors' contributions}

Study design by DTM, ADR, and GLS; medical records interpretation and data extraction by DTM, HA, RK, AS, DSC; data review and validation by MOS, ADR, and GS; data analysis by DTM and HA; major contributions to the writing and editing of the manuscript by DTM, HA, MOS, and GLS. All authors read and approved the final manuscript.

\section{Funding}

There were no sources of funding for this study.

\section{Availability of data and materials}

The datasets used and analyzed for this study are available from the corresponding author upon reasonable request. The diagnostic methods used are available from the manufacturer as specified in the text.

\section{Ethics approval and consent to participate}

Ethics approval for this retrospective study was provided by the GW University Office of Human Research Institutional Review Board (GW IRB). Consent for participation was waived as this was a retrospective review of medical records with no patient contact and no active medical intervention.

\section{Consent for publication}

Consent for publication was also waived per the GWU IRB.

\section{Competing interests}

The authors declare that they have no competing commercial or research interests for this study.

Received: 9 January 2020 Accepted: 22 July 2020

Published online: 29 July 2020

\section{References}

1. Farthing M, Salam MA, Lindberg G, Dite P, Khalif I, Salazar-Lindo E, et al. Acute diarrhea in adults and children: a global perspective. J Clin Gastroenterol. 2013;47:12-20. 
2. GBD Diarrhoeal Diseases Collaborators. Estimates of global, regional, and national morbidity, mortality, and aetiologies of diarrhoeal diseases: a systematic analysis for the Global Burden of Disease Study 2015. Lancet Infect Dis. 2017;17:909-48.

3. Kosek M, Bern C, Guerrant RL. The global burden of diarrhoeal disease, as estimated from studies published between 1992 and 2000. Bull World Health Organ. 2003;81:197-204.

4. Bresee JS, Marcus R, Venezia RA, Keene WE, Morse D, Thanassi M, et al. The etiology of severe acute gastroenteritis among adults visiting emergency departments in the United States. J Infect Dis. 2012;205:1374-81.

5. Jones TF, McMillian MB, Scallan E, Frenzen PD, Cronquist AB, Thomas S, Angulo FJ. A population-based estimate of the substantial burden of diarrhoeal disease in the United States; FoodNet, 1996-2003. Epidemiol Infect. 2007;135:293-301.

6. Scallan E, Griffin PM, Angulo FJ, Tauxe RV, Hoekstra RM. Foodborne illness acquired in the United States-unspecified agents. Emerg Infect Dis. 2011;17:16-22.

7. Myer PA, Mannalithara A, Singh G, Singh G, Pasricha PJ, Ladabaum U. Clinical and economic burden of emergency department visits due to gastrointestinal diseases in the United States. Am J Gastroenterol. 2013;108:1496-507.

8. Perez JM, Cavalli P, Roure C, Renac R, Gille Y, Freydiere AM. Comparison of four chromogenic media and Hektoen agar for detection and presumptive identification of Salmonella strains in human stools. J Clin Microbiol. 2003;41:1130-4.

9. Bennett WE, Tarr PI. Enteric infections and diagnostic testing. Curr Opin Gastroenterol. 2009;25:1-7.

10. Hatchette TF, Farina D. Infectious diarrhea: when to test and when to treat. CMAJ. 2011;183:339-44.

11. Hennessy TW, Marcus R, Deneen V, Reddy S, Vugia D, Townes J, Bardsley M, et al. Survey of physician diagnostic practices for patients with acute diarrhea: clinical and public health implications. Clin Infect Dis. 2004;38:S203-11.

12. Guarino A, Giannattasio A. New molecular approaches in the diagnosis of acute diarrhea: advantages for clinicians and researchers. Curr Opin Gastroenterol. 2011:27:24-9.

13. Amar CF, East CL, Gray J, Iturriza-Gomara M, Maclure EA, McLauchlin J. Detection by PCR of eight groups of enteric pathogens in 4,627 faecal samples. Eur J Clin Microbiol Infect Dis. 2007;26:311-23.

14. de Boer RF, Ott A, Kesztyüs B, Kooistra-Smid AM. Improved detection of five major gastrointestinal pathogens by use of a molecular screening approach. J Clin Microbiol. 2010;48:4140-6.

15. Halligan E, Edgeworth J, Bisnauthsing K, Bible J, Cliff P, Aarons E, et al. Multiplex molecular testing for management of infectious gastroenteritis in a hospital setting: a comparative diagnostic and clinical utility study. Clin Microbiol Infect. 2014;20:0460-7.

16. US Food and Drug Administration. 510(k) substantial equivalence determination decision summary K140407. 2014. http://www.accessdata.fda. gov/cdrh_docs/reviews/k140407.pdf. Accessed 26 Oct 2018.

17. Buss SN, Leber A, Chapin K, Fey PD, Bankowski MJ, Jones MK, et al. Multicenter evaluation of the BioFire FilmArray gastrointestinal panel for etiologic diagnosis of infectious gastroenteritis. J Clin Microbiol. 2015;53:915-25.

18. Freeman K, Tsertsvadze A, Taylor-Phillips S, McCarthy N, Mistry H, et al. Agreement between gastrointestinal panel testing and standard microbiology methods for detecting pathogens in suspected infectious gastroenteritis: test evaluation and meta-analysis in the absence of a reference standard. PLoS One. 2017. https://doi.org/10.1371/journal.pone.0173196.

19. Stockmann C, Rogatcheva M, Harrel B, Vaughn M, Crisp R, Poritz M, et al. How well does physician selection of microbiologic tests identify Clostridium difficile and other pathogens in paediatric diarrhoea? Insights using multiplex PCR-based detection. Clin Microbiol Infect. 2015. https://doi. org/10.1016/j.cmi.2014.07.011.

20. Humphrey JM, Ranbhise S, Ibrahim E, Al-Romaihi HE, Farag E, et al. Multiplex polymerase chain reaction for detection of gastrointestinal pathogens in migrant workers in Qatar. Am J Trop Med Hyg. 2016;95:1330-7.

21. Becker SL, Chatigre JK, Gohou JP, Coulibaly JT, Leuppi R, Polman K, et al. Combined stool-based multiplex PCR and microscopy for enhanced pathogen detection in patients with persistent diarrhoea and asymptomatic controls from Côte d'Ivoire. Clin Microbiol Infect. 2015. https://doi.org/10. 1016/j.cmi.2015.02.016.

22. Spina A, Kerr KG, Cormican M, Barbut F, Eigentler A, Zerva L, et al. Spectrum of enteropathogens detected by the FilmArray GI Panel in a multicentre study of community-acquired gastroenteritis. Clin Microbiol Infect. 2015;21:719-28.

23. Khare R, Espy MJ, Cebelinski E, Boxrud D, Sloan LM, Cunningham SA, et al. Comparative evaluation of two commercial multiplex panels for detection of gastrointestinal pathogens by use of clinical stool specimens. J Clin Microbiol. 2014;52:3667-73.

24. Zhang H, Morrison S, Tang YW. Multiplex polymerase chain reaction tests for detection of pathogens associated with gastroenteritis. Clin Lab Med. 2015;35:461-86.

25. Piralla A, Lunghi G, Ardissino G, Girello A, Premoli M, Bava E, et al. FilmArray Gl panel performance for the diagnosis of acute gastroenteritis or hemorragic diarrhea. BMC Microbiol. 2017;17:111.

26. Freeman K, Mistry H, Tsertsvadze A, Royle P, McCarthy N, Taylor-Phillips S, et al. Multiplex tests to identify gastrointestinal bacteria, viruses and parasites in people with suspected infectious gastroenteritis: a systematic review and economic analysis. Health Technol Assess. 2017;21:1-188.

27. Murphy CN, Fowler RC, Iwen PC, Fey PD. Evaluation of the BioFire FilmArray ${ }^{\oplus}$ gastrointestinal panel in a Midwestern academic hospital. Eur J Clin Microbiol Infect Dis. 2017;36:747-54.

28. Beal SG, Tremblay EE, Toffel S, Velez L, Rand KH. A gastrointestinal PCR panel improves clinical management and lowers health care costs. J Clin Microbiol. 2017. https://doi.org/10.1128/JCM.01457-17.

29. Goldenberg SD, Bacelar M, Brazier P, Bisnauthsing K, Edgeworth JD. A cost benefit analysis of the Luminex xTAG gastrointestinal pathogen panel for detection of infectious gastroenteritis in hospitalised patients. J Inf Secur. 2015;70:504-11.

30. Rand KH, Tremblay EE, Hoidal M, Fisher LB, Grau KR, Karst SM. Multiplex gastrointestinal pathogen panels: implications for infection control. Diagn Microbiol Infect Dis. 2015;82:154-7.

31. Binnicker MJ. Multiplex molecular panels for diagnosis of gastrointestinal infection: performance, result interpretation, and cost-effectiveness. J Clin Microbiol. 2015;53:3723-8.

32. Hitchcock MM, Gomez CA, Banaei N. Low yield of FilmArray GI panel in hospitalized patients with diarrhea: an opportunity for diagnostic stewardship intervention. J Clin Microbiol. 2018. https://doi.org/10.1128/JCM.01558-17.

\section{Publisher's Note}

Springer Nature remains neutral with regard to jurisdictional claims in published maps and institutional affiliations.

\section{Ready to submit your research? Choose BMC and benefit from:}

- fast, convenient online submission

- thorough peer review by experienced researchers in your field

- rapid publication on acceptance

- support for research data, including large and complex data types

- gold Open Access which fosters wider collaboration and increased citations

- maximum visibility for your research: over $100 \mathrm{M}$ website views per year

At BMC, research is always in progress.

Learn more biomedcentral.com/submissions 